\title{
Probing the Spatiotemporal Dynamics of Catalytic Janus Particles with Single-Particle Tracking and Differential Dynamic Microscopy
}

\author{
Christina Kurzthaler, ${ }^{1}$ Clémence Devailly, ${ }^{2}$ Jochen Arlt, ${ }^{2}$ Thomas Franosch, ${ }^{1}$ Wilson C. K. Poon, ${ }^{2}$ \\ Vincent A. Martinez, ${ }^{2, *}$ and Aidan T. Brown ${ }^{2, \dagger}$ \\ ${ }^{1}$ Institut für Theoretische Physik, Universität Innsbruck, Technikerstraße 21A, A-6020 Innsbruck, Austria \\ ${ }^{2}$ School of Physics and Astronomy, University of Edinburgh, \\ James Clerk Maxwell Building, Peter Guthrie Tait Road, Edinburgh EH9 3FD, United Kingdom
}

(Received 7 December 2017; revised manuscript received 1 May 2018; published 14 August 2018)

\begin{abstract}
We demonstrate differential dynamic microscopy and particle tracking for the characterization of the spatiotemporal behavior of active Janus colloids in terms of the intermediate scattering function (ISF). We provide an analytical solution for the ISF of the paradigmatic active Brownian particle model and find striking agreement with experimental results from the smallest length scales, where translational diffusion and self-propulsion dominate, up to the largest ones, which probe effective diffusion due to rotational Brownian motion. At intermediate length scales, characteristic oscillations resolve the crossover between directed motion to orientational relaxation and allow us to discriminate active Brownian motion from other reorientation processes, e.g., run-and-tumble motion. A direct comparison to theoretical predictions reliably yields the rotational and translational diffusion coefficients of the particles, the mean and width of their speed distribution, and the temporal evolution of these parameters.
\end{abstract}

DOI: 10.1103/PhysRevLett.121.078001

Microswimmers exhibit a vast variety of propulsion mechanisms, ranging from turning helical screws in bacteria [1] to the phoretic motion of synthetic, patterned (Janus) particles [2-6]. These self-propelled agents are intrinsically out of equilibrium and display peculiar dynamical behavior due to the interplay of persistent swimming motion and stochastic fluctuations [7-11]. An intriguing feature displayed by microswimmers is the randomization of their swimming motion at large length scales due to various reorientation mechanisms, e.g., rotational diffusion expected for catalytic Janus colloids, or the "tumbling" typical of flagellated bacteria. These reorientation processes are significant for both collective dynamics $[12,13]$ and microbiology, where many organisms achieve chemotaxis by varying their tumbling rate $[1,14]$.

Experimental techniques for characterizing microswimmer behavior can be classified broadly into singleparticle tracking [3] and ensemble techniques like dynamic light scattering $[5,15]$ or differential dynamic microscopy (DDM) $[16,17]$. These approaches are complementary, with different merits and drawbacks. Single-particle tracking provides direct access to individual trajectories and therefore full statistical information. However, tracking requires

Published by the American Physical Society under the terms of the Creative Commons Attribution 4.0 International license. Further distribution of this work must maintain attribution to the author(s) and the published article's title, journal citation, and DOI. optical resolution of single particles, and tracking of bulk (3D) systems is challenging because particles disappear from the image plane. 3D tracking is possible using Lagrangian microscopes [1,18] or holographic microscopy [19], but these techniques are limited in statistical accuracy or limited to low particle concentration, respectively.

In contrast, DDM provides high-throughput measurements of the intermediate scattering function (ISF) [16,17]. It is suitable for low-resolution microscopy with a large field of view, so can access large length scales, and is not restricted to low particle concentration [20,21]. DDM usually gives the ensemble-averaged dynamics over entire populations, so that using DDM to extract information on single-particle dynamics is nonideal. For noninteracting particles, the ISF constitutes the Fourier transform of the probability density of the particle displacements and encodes full statistical information about particle behavior at a given length scale $l$ and delay time $\tau$. Hence, a quantitative comparison of experimental ISFs to theoretical predictions over a broad range of $l$ and $\tau$ should permit full characterization of the motion and extraction of key dynamical quantities.

To date, the ISF of self-propelled particles has only been measured, via DDM, at relatively small length scales for which directed motion dominates the dynamics [17,20,22-26]. Larger length scales over which reorientation leads to random motion have not been accessed yet. Theoretical predictions for the ISF of active agents have been elaborated recently probing different modes of reorientation. In particular, analytical expressions of the ISF have been obtained for the paradigmatic active 
Brownian particle (ABP) model in three dimensions [27], where the orientation undergoes rotational diffusion, and for run-and-tumble particles (RTPs) in two dimensions $[28,29]$, which perform instantaneous, temporally uncorrelated tumbling events.

In this Letter, we use DDM and particle tracking to characterize the spatiotemporal behavior of self-propelled catalytic Janus particles at a glass surface. We measure the ISF over a wide range of length scales and timescales and show that characteristic features emerge at intermediate length scales, where orientational relaxation occurs. These features allow us to discriminate between different propulsion models. Fitting of the ISFs using the ABP model allows extraction of key dynamical quantities; i.e., the translational $(D)$ and rotational $\left(D_{\text {rot }}\right)$ diffusion coefficients; and the mean $\langle v\rangle$ and width $\sigma_{v}$ of the swimming speed distribution.

Experimental method.-Self-propelled Janus colloids [3] were manufactured by sputter coating $r=0.96 \pm 0.04 \mu \mathrm{m}$ radius green fluorescent polystyrene colloids (Invitrogen) with a 5-nm-thick hemispherical Pt shell [6,30]. We suspended the Janus colloids at volume fraction $10^{-5}$ in $0.75 \% \mathrm{w} / \mathrm{w}$ aqueous $\mathrm{H}_{2} \mathrm{O}_{2}$ solutions (Acros) and placed this suspension in chambers assembled from glass microscopy slides (Menzel) and $22 \times 22 \mathrm{~mm}^{2}$ glass coverslips (Bettering) with $\sim 300 \mu \mathrm{m}$ Parafilm spacers. The Janus particles self-propel by decomposing the $\mathrm{H}_{2} \mathrm{O}_{2}$ on their $\mathrm{Pt}$ face [3], though the detailed propulsion mechanism remains uncertain $[6,31,32]$. The particles are bottom heavy [33], so swim towards the upper surface of the chamber, and then slide stably along that surface oriented approximately parallel to the surface in a quasi-2D layer [6,34]. We captured a 40 min-long sequence of epifluorescence images $(512 \times 512$ pixels) at $50 \mathrm{fps}$ (exposure time $T_{e}=0.02 \mathrm{~s}$ ), consisting of 15 submovies of $\approx 8000$ images, using an inverted microscope ( $\mathrm{Ti}$ Eclipse, Nikon) and low magnification objective (Nikon Plan Fluor 10xPh1, NA $=0.3$ ) with a sCMOS camera (Hamamatsu Orca-Flash 4.0) and Micromanager [35]. Images were recorded with $4 \times 4$ pixel binning giving a $2.6 \mu \mathrm{m} /$ pixel resolution, at $23-25^{\circ} \mathrm{C}$, and focusing on the upper glass surface. The number of particles per field of view increases from $\approx 60$ to $\approx 90$ particles after $15 \mathrm{~min}$.

The time sequence of images was analyzed via DDM [17,23] and standard particle tracking algorithms [6,30]. From particle tracking, the ISFs of the particles were computed via $f(k, \tau)=\left\langle e^{-\mathbf{i k} \cdot \Delta \mathbf{r}_{j}(\tau)}\right\rangle$, with $\Delta \mathbf{r}_{j}(\tau)$ the displacement of particle $j$ at lag time $\tau, \mathbf{k}$ the wave vector with magnitude $k=|\mathbf{k}|$ probing the dynamics at length scale $l=2 \pi / k$, and brackets $\langle\cdot\rangle$ denoting averages over all particles $j$ and orientations of the wave vector. A fraction of tracks displaying "nonideal" behavior (i.e., getting stuck to glass or swimming away from the glass) were discarded resulting in $\approx 60$ "well-behaved" tracks ( $\gtrsim 120 \mathrm{~s}$ long) of particles at the surface. For DDM analysis, the differential image correlation function, $g(\mathbf{k}, \tau)$, i.e., the time-averaged power spectrum of the difference between pairs of images separated by time $\tau$, was computed, $g(\mathbf{k}, \tau)=\langle| I(\mathbf{k}, t+\tau)-$ $\left.\left.I(\mathbf{k}, t)\right|^{2}\right\rangle_{t}$, with $I(\mathbf{k}, t)$ the Fourier transform of the image $I(\mathbf{r}, t)$. Under appropriate imaging conditions and for isotropic motion [17,23], $g(k, \tau)=A(k)[1-f(k, \tau)]+B(k)$, with $A(k)$ the signal amplitude and $B(k)$ the camera noise. $A(k)$ is obtained from the long-time limit of $g(k, \tau)$, where $f(k, \tau) \rightarrow 0$, while in the present case of epifluorescence imaging the camera noise is negligible [36].

Theory.-The motion of an ABP in two dimensions consists of isotropic translational diffusion with coefficient $D$ and directed self-propulsion at a fixed speed $v$ along the particle's instantaneous orientation $\mathbf{u}=(\cos \vartheta, \sin \vartheta)$, which undergoes rotational diffusion with coefficient $D_{\text {rot }}$. Thus, an ABP's trajectory displays a characteristic persistence length $L=v / D_{\text {rot }}$. We describe the ABP model in two dimensions using a Fokker-Planck equation [45] (for the Langevin equations see the Supplemental Material Ref. [36])

$$
\partial_{\tau} \mathbb{P}=-v \mathbf{u} \cdot \nabla_{\mathbf{r}} \mathbb{P}+D_{\text {rot }} \partial_{\vartheta}^{2} \mathbb{P}+D \nabla_{\mathbf{r}}^{2} \mathbb{P},
$$

with $\mathbb{P}\left(\Delta \mathbf{r}, \vartheta, \tau \mid \vartheta_{0}\right)$ the probability density for an ABP to undergo a displacement $\Delta \mathbf{r}$ and reorient from an initial angle $\vartheta_{0}$ to a final angle $\vartheta$ in time $\tau$, and $\nabla_{\mathbf{r}}$ the spatial gradient. The terms on the right-hand side correspond to propulsion, rotational, and translational diffusion, respectively. The ISF is obtained by a spatial Fourier transform, $\tilde{\mathbb{P}}\left(\mathbf{k}, \vartheta, \tau \mid \vartheta_{0}\right)=\int d^{2} r \exp (-\mathrm{i} \mathbf{k} \cdot \mathbf{r}) \mathbb{P}\left(\mathbf{r}, \vartheta, \tau \mid \vartheta_{0}\right)$, averaged over $\vartheta_{0}$ and integrated over $\vartheta$

$$
f(k, \tau)=\left\langle e^{-i \mathbf{k} \cdot \Delta \mathbf{r}(\tau)}\right\rangle=\int d \vartheta \int \frac{d \vartheta_{0}}{2 \pi} \tilde{\mathbb{P}}\left(\mathbf{k}, \vartheta, \tau \mid \vartheta_{0}\right) .
$$

The equation of motion for $\tilde{\mathbb{P}}$ is solved by following a similar solution strategy as for an ABP in three dimensions [27], by separating variables in terms of angular eigenfunctions [36]. This yields an exact expression for the ISF

$$
f(k, \tau)=e^{-k^{2} D \tau} \sum_{n=0}^{\infty} e^{-\lambda_{2 n} \tau}\left(\int_{0}^{2 \pi} \frac{d \vartheta}{2 \pi} \operatorname{ce}_{2 n}(q, \vartheta / 2)\right)^{2},
$$

where the even, $\pi$-periodic Mathieu functions $\operatorname{ce}_{2 n}(q, \vartheta)$ [46] have imaginary deformation parameter $q=2 \mathrm{i} k L$ and $\lambda_{2 n}=a_{2 n}(q) D_{\text {rot }} / 4$ with $a_{2 n}(q)$ the eigenvalues of the Mathieu functions.

For comparison, the motion of a RTP is characterized by straight-run phases interrupted by instantaneous tumbling events that randomize the swimming direction. The tumbling events are exponentially distributed with rate $\lambda$ [see Ref. [29], Eq. (1)]. The ISF of a 2D RTP is [29]

$$
f(k, \tau)=e^{-k^{2} D \tau} \sum_{n=0}^{\infty} \frac{e^{-\lambda \tau} \sqrt{\pi}}{\Gamma[(n+1) / 2]}\left(\frac{\lambda^{2} \tau}{2 k v}\right)^{n / 2} J_{n / 2}(k v \tau),
$$

with $J_{n / 2}(\cdot)$ the Bessel function of order $n / 2$. The ISFs for ABPs [Eq. (3)] and RTPs [Eq. (4)] share identical forms in 
the large and small $k$ regimes, where the reorientation mechanisms have not yet set in or are no longer resolved (see the Supplemental Material [36]). In particular, both ISFs can be approximated to $f(k, \tau) \approx$ $J_{0}(v k \tau) \exp \left(-D k^{2} \tau\right)$ at short times, $\tau \lesssim \tau_{\text {rot }}:=1 / D_{\text {rot }}$ and $\tau \lesssim 1 / \lambda$ for ABPs and RTPs, respectively. At these small times and large wave numbers $k D / v \gtrsim 1, J_{0}(v k \tau) \rightarrow 1$ and $f(k, \tau) \approx \exp \left(-D k^{2} \tau\right)$. In contrast, for intermediate wave numbers, characteristic oscillations emerge due to the swimming motion encoded in the Bessel function. At long times and small wave numbers $k L \ll 1, \quad f(k, \tau) \approx$ $\exp \left(-D_{\text {eff }} k^{2} \tau\right)$ with effective diffusion $D_{\text {eff }}=v^{2} / 2 D_{\text {rot }}+$ $D$ for ABPs or with $D_{\text {rot }}$ replaced by $\lambda$ for RTPs. At $k$ values probing the reorientation mechanisms, $k L \simeq 2 \pi$, the two ISFs are expected to display distinct behaviors reflecting rotational diffusion or random tumbling, respectively. Thus, precise measurement of the ISF at intermediate length scales should distinguish between ABPs and RTPs. By contrast, the mean-square displacements (MSD) for these two models and a range of similar models, e.g., particles simultaneously exhibiting rotational diffusion and tumbling, are identical for all lag times $[29,47]$.

Results.-Fig. 1(a) shows the ISF of catalytic Janus particles measured via DDM for a large window of wave numbers and lag times. As predicted by the ABP model, the ISF exhibits oscillations at intermediate times and wave numbers $k \gtrsim 0.3 \mu \mathrm{m}^{-1}$, which fall off for $\tau \gtrsim 4 \mathrm{~s}$ due to rotational diffusion and evolve to an effective diffusive behavior for small $k \lesssim 0.3 \mu \mathrm{m}^{-1}$; at the highest $k \approx 1.2 \mu \mathrm{m}^{-1}$, translational diffusion begins to damp out the oscillations. Fitting of the experimental ISFs using Eq. (3) and considering a Gaussian speed distribution $P(v)$, with mean $\langle v\rangle$ and width $\sigma_{v}$, shows good quantitative agreement over all time and length scales considered. The largest amplitude peak at $k=0.71 \mu \mathrm{m}^{-1}$ corresponds to approximately twice the persistence length, $L=\langle v\rangle / D_{\text {rot }} \simeq 5 \mu \mathrm{m}$, characterizing the transition from directed motion to effective diffusion.

Part of $\sigma_{v}$ comes from particles slowing down due to $\mathrm{H}_{2} \mathrm{O}_{2}$ consumption. Analysis of short sections of the original video reveals a time dependence in the mean propulsion speed $\langle v\rangle$, Fig. 1(b), consistent with the exponential decay previously obtained in reaction-rate measurements on the same system [6]. We estimate that over $40 \mathrm{~min}$ this exponential decay should contribute $\approx 0.1 \mu \mathrm{ms}^{-1}$ to the measured standard deviation. The standard deviation itself would also be expected to decay exponentially, by about $20 \%$, but this is smaller than the noise in this parameter [inset Fig. 1(b)]. $D$ (not shown) is constant within error, and an average over the experimental time window gives $D=0.233 \pm 0.003 \mu \mathrm{m}^{2} \mathrm{~s}^{-1}$. The apparent time dependence of $D_{\text {rot }}$ is due to a few bright, actively rotating particles present at short times; cropping the early videos to remove these features reduces this effect significantly, Fig. 1(c) [36].

The computed ISFs from single-particle tracks show very good agreement with the ISFs obtained from DDM [see Fig. 1(d)]. The deviations at $k=0.12 \mu \mathrm{m}^{-1}$ at long times, where no reliable plateau is observed, can be solely traced back to noise in the data, since the delay times here are almost equal to the length of each submovie $\sim 160 \mathrm{~s}$. Parameter estimation of the submovies reveals that the speed and the rotational diffusion coefficient (following cropping) agree reasonably between DDM and tracking [Figs. 1(b)-1(c)]. We find $D_{\text {rot }}=0.226 \pm 0.008 \mathrm{~s}^{-1}$ and $0.202 \pm 0.003 \mathrm{~s}^{-1}$ from DDM and tracking, respectively. Separately applying a "windowing" correction [48], which aims to correct for DDM artifacts introduced by particles moving out of the field of view, yielded $D_{\text {rot }}=$ $0.206 \pm 0.012 \mathrm{~s}^{-1}$, with other parameters left largely unchanged but more noisy [36].
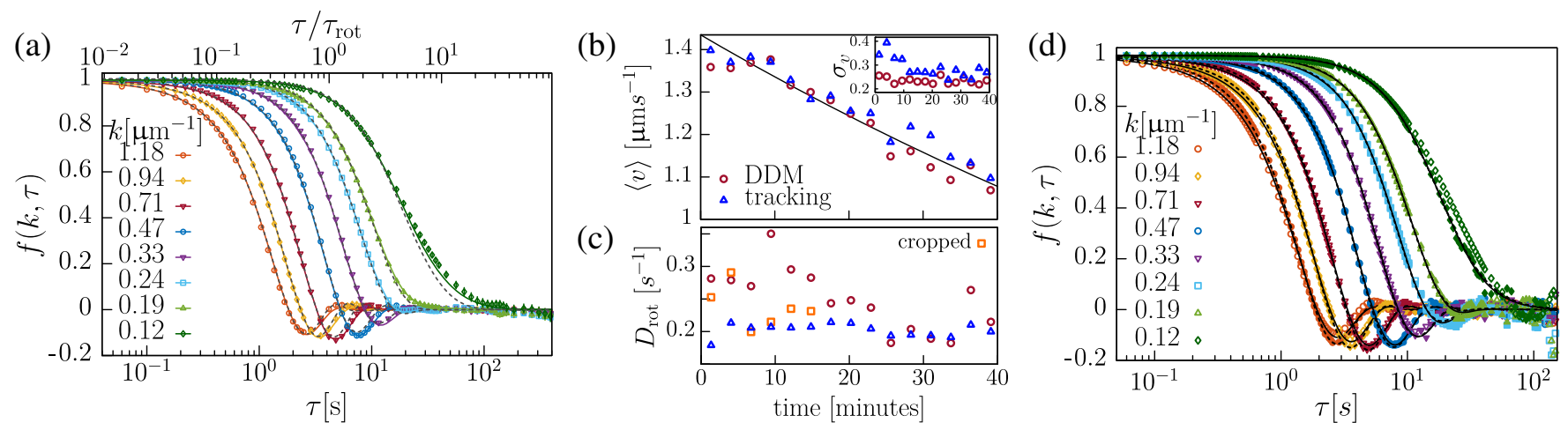

FIG. 1. (a) ISFs of a dilute suspension of Janus particles obtained via DDM (symbols and error bars) over the whole time sequence $\left(1.2 \times 10^{5}\right.$ images), fitted with the ABP model (colored lines) and the RTP model (dashed, gray lines), both averaged over a Gaussian speed distribution. $\tau_{\text {rot }}=D_{\text {rot }}^{-1}$ denotes the rotational diffusion time. Global fitting of the ISFs [36] using the ABP model yields $\langle v\rangle=1.23 \pm 0.01 \mu \mathrm{ms}^{-1}, \sigma_{v}=0.26 \pm 0.02 \mu \mathrm{ms}^{-1}, D_{\text {rot }}=0.24 \pm 0.01 \mathrm{~s}^{-1}$, and $\mathrm{D}=0.24 \pm 0.02 \mu \mathrm{m}^{2} \mathrm{~s}^{-1}$. Separately fitting the RTP model provides the tumbling rate $\lambda=0.20 \pm 0.03 \mathrm{~s}^{-1}$, and $\langle v\rangle=1.21 \pm 0.03 \mu \mathrm{m} \mathrm{s}^{-1}, \sigma_{v}=0.05 \pm 0.01 \mu \mathrm{m} \mathrm{s}{ }^{-1}, D=$ $0.26 \pm 0.02 \mu \mathrm{m}^{2} \mathrm{~s}^{-1}$. (b)-(c) Temporal variation of the motility parameters obtained from the ABP model fit of the ISFs extracted from DDM and tracking of fifteen 160 s submovies. The black line is $\propto \exp (-t / T)$ with $T=140$ min. (d) ABP-fitted ISFs from DDM (hollow symbols, solid line) and tracking (solid symbols, dashed line) for the submovie at $25 \mathrm{~min}$. 
By contrast, we observe a systematic deviation of the average translational diffusion coefficient between tracking $\left(D=0.172 \pm 0.002 \mu \mathrm{m}^{2} \mathrm{~s}^{-1}\right)$ and DDM $(D=0.233 \pm$ $0.003 \mu \mathrm{m}^{2} \mathrm{~s}^{-1}$ ). This difference can partially be explained by the presence of out-of-focus particles in the bulk, which have a higher diffusivity. Removing some of these out-offocus particles (by thresholding prior to DDM analysis) reduces the effect, giving $D=0.207 \pm 0.003 \mu \mathrm{m}^{2} \mathrm{~s}^{-1}$ for DDM.

The measured $D_{\text {rot }}$ agrees with the Stokes-Einstein prediction for equivalent equilibrium particles in the bulk, $D_{\text {rot }}^{\mathrm{SE}}=k_{B} T /\left(8 \pi \eta r^{3}\right)=0.21 \pm 0.03 \mathrm{~s}^{-1}$ with solution viscosity $\eta=0.91 \pm 0.02 \mathrm{mPas}$ estimated from literature values $[49,50]$. However, $D$ is lower than the bulk free diffusion coefficient of an equivalent passive colloid $D^{\mathrm{SE}}=$ $k_{B} \mathrm{~T} /(6 \pi \eta r)=0.25 \pm 0.02 \mu \mathrm{m}^{2} \mathrm{~s}^{-1}$. Proximity to the wall will likely account for some of this difference, just as for a passive colloid [51], for which one indeed expects only translational diffusion to be significantly perturbed [52].

Unlike the MSD, the ISF should allow us to discriminate between rotational diffusion and run-and-tumble motion. However, we see in Fig. 1(a) that the RTP model also reproduces surprisingly well the main features of the experimental ISFs. This is because of the qualitative similarity between the ABP and RTP ISFs, and because the finite width of the speed distribution $P(v)$, inherently present in the swimmer population, gives an additional degree of freedom through the fitting parameter $\sigma_{v}$. We also separately fitted each submovie, which removes the temporal part of the speed distribution (for $160 \mathrm{~s}$ segments, the expected temporal contribution is only $7 \mathrm{~nm} \mathrm{~s}^{-1}$ ), enhancing the distinction between the swimming mechanisms. Significantly better agreement with the ABP model is obtained for all submovies: the mean-squared error (MSE), for the ABP model is $(1.02 \pm 0.09) \times 10^{-4}$, whereas for the RTP model, it is roughly $50 \%$ larger at $(1.53 \pm 0.08) \times 10^{-4}$.

To strengthen these results, we investigated the ISF computed from single-particle tracks, e.g., Fig. 2, recorded at higher magnification (x20 Nikon Plan Apo Ph1, $\mathrm{NA}=0.75,1 \times 1$ binning, $351 \mathrm{~nm} /$ pixel) to give access to higher $k$ values. Fitting the ISFs with the ABP and RTP models (now with a single speed $v$ ) reveals small deviations at short times $\left(\tau<0.01 \tau_{\text {rot }}\right)$ for $k \gtrsim 2.3 \mu \mathrm{m}^{-1}$, but correcting for the effect of the finite exposure time $T_{e}$ removes these deviations (Fig. 2 and the Supplemental Material [36]).

As expected, both ABP and RTP models agree at short times where the swimming direction has not changed due to rotational diffusion or instantaneous tumbling events. For $\tau \lesssim \tau_{\text {rot }}$ and large $k$, oscillations are strongly damped by translational diffusion, tending towards a single exponential decay. Deviations between the ABP and RTP models become apparent for $\tau \simeq \tau_{\text {rot }}$ and $k L \simeq 2 \pi$ (in Fig. 2: $k \simeq 0.80 \mu \mathrm{m}^{-1}$ ), where the underlying reorientation mechanisms determine the particle dynamics (plotting the fitting

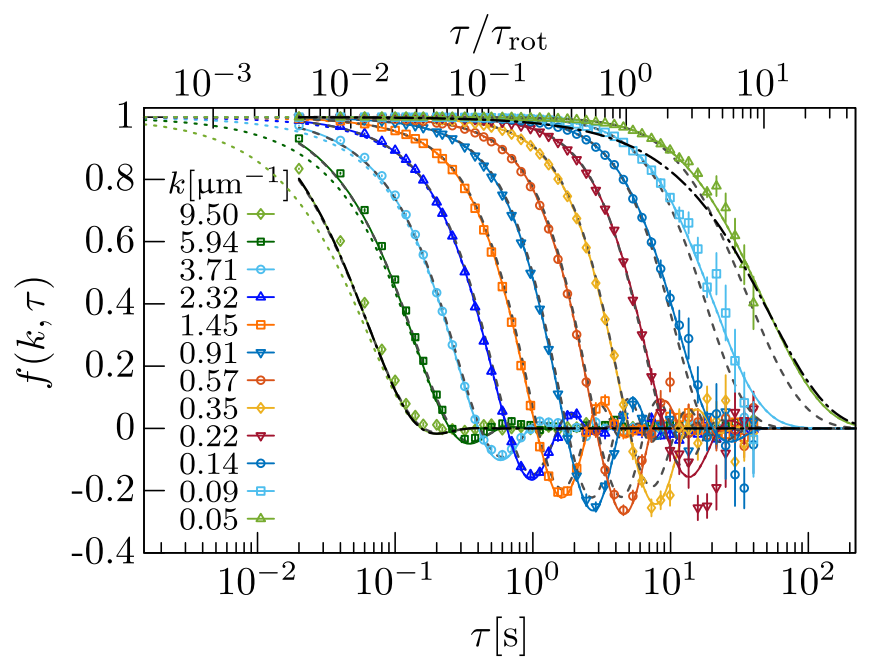

FIG. 2. ISFs for a single Janus particle (symbols and error bars, as in Fig. 1). Colored solid lines correspond to the theoretical predictions for ABPs $(v=1.64 \pm 0.02 \mu \mathrm{m} / \mathrm{s}$, $D=0.16 \pm 0.01 \mu \mathrm{m}^{2} / \mathrm{s}$, and $\left.D_{\text {rot }}=0.21 \pm 0.02 \mathrm{~s}^{-1}\right)$ and gray dashed lines for equivalent RTPs $(v=1.58 \pm 0.02 \mu \mathrm{m} / \mathrm{s}$, $D=0.17 \pm 0.01 \mu \mathrm{m}^{2} / \mathrm{s}$, and $\lambda=0.14 \pm 0.01 \mathrm{~s}^{-1}$ ), both corrected for a finite exposure time $T_{e}$. Colored dashed lines correspond to the uncorrected ABP ISF [Eq. (3)]. Dash-dotted, black lines indicate the corrected large and uncorrected small wave number approximations, $\exp \left[-k^{2} D\left(\tau-T_{e} / 3\right)\right] J_{0}(v k \tau)$ and $\exp \left(-k^{2} D_{\text {eff }} \tau\right)$, respectively. Note that the ISF does not approach the small wave number approximation closely even at the smallest $k$ displayed, for which $k L=0.43$.

residuals highlights these deviations, see the Supplemental Material [36]). These results confirm the systematic small quantitative difference between the RTP model and the experimental data observed in DDM, while the ABP model closely reproduces the experimental ISFs over 2 and 3 decades of length scales and timescales, respectively. We repeated this analysis with, in total, 23 "well-behaved" single-particle trajectories; in all but 4 of these, the $\mathrm{ABP}$ model reproduced the data better, with $\mathrm{MSE}=$ $(4.43 \pm 1.03) \times 10^{-4}$, than the RTP model, MSE $=$ $(5.46 \pm 0.65) \times 10^{-4}$ (averaged over all 23 trajectories, and for $\tau \lesssim 10 \mathrm{~s}$ ).

Summary and conclusion.-We have characterized the spatiotemporal dynamics of hydrogen-peroxide-fueled Janus colloids using DDM and particle tracking. Experimental observations of the ISF showed striking agreement with theoretical predictions of the active Brownian particle model over a broad range of length scales and timescales, reflecting the transition from directed swimming motion to the randomization of the orientation. The ISFs allowed us to distinguish between different modes of orientational relaxation (continuous rotational diffusion versus instantaneous tumbling) by probing the dynamics directly at the relevant length scale, i.e., the persistence length of the active agents. Additionally, we have demonstrated DDM as a high-throughput method to extract 
relevant motility parameters (and their temporal variation) of ensembles of catalytic Janus colloids.

Most previous studies of active colloids focused on the MSD obtained from single-particle tracking [3,4,6,30,31, 34,47,53], which does not discriminate between ABPs, RTPs, or similar models, in contrast to the ISF. One prior study measured higher moments of the particle motion, e.g., the non-Gaussian parameter, which should differentiate between different types of orientational relaxation [54], but comparison with the ABP model yielded no quantitative agreement there. This might result from statistical uncertainties or from, e.g., anisotropic translational diffusion [27].

Here, we showed that the standard ABP model fully describes the dynamics of phoretically driven Janus colloids even at small length scales $l$ that go down to a fraction of the particle diameter, i.e., $l / 2 r \approx 0.35$ (for $\left.\mathrm{k}=9.5 \mu \mathrm{m}^{-1}\right)$. This indicates that microscopic details of the propulsion mechanism [6,32,55-57] can be coarse grained into a few minimal, mesoscopic processes: propulsion, rotational diffusion, and translational diffusion, discussed here, as well as anisotropic diffusion [27] and deterministic rotational drift [58], which are relevant for anisotropic or chiral Janus particles $[47,59,60]$ and swimming bacteria near walls $[61,62]$.

In particular, our results show that extensions to the $\mathrm{ABP}$ model to include, e.g., temporal variations in particle speed [63] are not necessary. Such effects have been hypothesized to arise from hydrodynamic and other interactions between the active particle and the surface leading to, e.g., spontaneous oscillations [64,65] and thermal fluctuations in particle orientation with respect to the surface [54]. Though our results show that these effects do not significantly perturb the ABP behavior within our experimental window, probing these effects at shorter timescales and/or length scales remains an interesting research avenue.

We anticipate that our results will serve as a reference for the dynamics of synthetic self-propelled particles irrespective of their propulsion mechanism. Moreover, DDM provides a powerful tool to probe the dynamics of microswimmers over large length scales, and overcomes the statistical limitations of 3D single-particle tracking and poor optical resolution. Therefore, it can be efficiently applied to investigate the spatiotemporal dynamics of active particles moving in three dimensions $[24,25,66]$ or dense suspensions [67-69], or of smaller size [5,70]. In particular, DDM might shed light on the self-propulsion of enzymebased nanomotors [71,72] in the presence of strong stochastic forces, the run-and-tumble behavior of biological microswimmers, such as E. coli bacteria [1], or the chemotactic response of synthetic vesicles, which have potential for drug delivery [73].

The research data presented in this publication are available on the Edinburgh DataShare repository [74].
We thank Benno Liebchen and Sebastian Leitmann for helpful and stimulating discussions. This work was supported by the Austrian Science Fund (FWF) P 28687-N27, the UK EPSRC Grant No. EP/J007404/1, and the ERC Advanced Grant No. ERC-2013-AdG 340877-PHYSAP.

*vincent.martinez@ed.ac.uk †abrown20@staffmail.ed.ac.uk

[1] H. C. Berg and D. A. Brown, Nature (London) 239, 500 (1972).

[2] W. F. Paxton, K. C. Kistler, C. C. Olmeda, A. Sen, S. K. St. Angelo, Y. Cao, T. E. Mallouk, P. E. Lammert, and V. H. Crespi, J. Am. Chem. Soc. 126, 13424 (2004).

[3] J. R. Howse, R. A. L. Jones, A. J. Ryan, T. Gough, R. Vafabakhsh, and R. Golestanian, Phys. Rev. Lett. 99, 048102 (2007).

[4] H.-R. Jiang, N. Yoshinaga, and M. Sano, Phys. Rev. Lett. 105, 268302 (2010).

[5] T.-C. Lee, M. Alarcón-Correa, C. Miksch, K. Hahn, J. G. Gibbs, and P. Fischer, Nano Lett. 14, 2407 (2014).

[6] A. T. Brown and W. C. K. Poon, Soft Matter 10, 4016 (2014).

[7] T. Vicsek and A. Zafeiris, Phys. Rep. 517, 71 (2012).

[8] P. Romanczuk, M. Bär, W. Ebeling, B. Lindner, and L. Schimansky-Geier, Eur. Phys. J. Spec. Top. 202, 1 (2012).

[9] M. C. Marchetti, J. F. Joanny, S. Ramaswamy, T. B. Liverpool, J. Prost, M. Rao, and R. A. Simha, Rev. Mod. Phys. 85, 1143 (2013).

[10] J. Elgeti, R. G. Winkler, and G. Gompper, Rep. Prog. Phys. 78, 056601 (2015).

[11] C. Bechinger, R. Di Leonardo, H. Löwen, C. Reichhardt, G. Volpe, and G. Volpe, Rev. Mod. Phys. 88, 045006 (2016).

[12] M. E. Cates and J. Tailleur, Europhys. Lett. 101, 20010 (2013).

[13] M. Khatami, K. Wolff, O. Pohl, M. R. Ejtehadi, and H. Stark, Sci. Rep. 6, 37670 (2016).

[14] J. Taktikos, H. Stark, and V. Zaburdaev, PLoS One 8, e81936 (2013).

[15] B. J. Berne and R. Pecora, Dynamic Light Scattering (John Wiley and Sons, New York, 1976).

[16] R. Cerbino and V. Trappe, Phys. Rev. Lett. 100, 188102 (2008).

[17] L. G. Wilson, V. A. Martinez, J. Schwarz-Linek, J. Tailleur, G. Bryant, P. N. Pusey, and W. C. K. Poon, Phys. Rev. Lett. 106, 018101 (2011).

[18] T. Darnige, N. Figueroa-Morales, P. Bohec, A. Lindner, and E. Clément, Rev. Sci. Instrum. 88, 055106 (2017).

[19] J. Sheng, E. Malkiel, and J. Katz, Appl. Opt. 45, 3893 (2006).

[20] J. Schwarz-Linek, J. Arlt, A. Jepson, A. Dawson, T. Vissers, D. Miroli, T. Pilizota, V. A. Martinez, and W. C. K. Poon, Colloids Surf. B 137, 2 (2016).

[21] T. Sentjabrskaja, E. Zaccarelli, C. De Michele, F. Sciortino, P. Tartaglia, T. Voigtmann, S. U. Egelhaaf, and M. Laurati, Nat. Commun. 7, 11133 (2016).

[22] P. J. Lu, F. Giavazzi, T. E. Angelini, E. Zaccarelli, F. Jargstorff, A. B. Schofield, J. N. Wilking, M. B. Romanowsky, D. A. Weitz, and R. Cerbino, Phys. Rev. Lett. 108, 218103 (2012). 
[23] V. A. Martinez, R. Besseling, O. A. Croze, J. Tailleur, M. Reufer, J. Schwarz-Linek, L. G. Wilson, M. A. Bees, and W. C. K. Poon, Biophys. J. 103, 1637 (2012).

[24] A. Jepson, V. A. Martinez, J. Schwarz-Linek, A. Morozov, and W. C. K. Poon, Phys. Rev. E 88, 041002 (2013).

[25] V. A. Martinez, J. Schwarz-Linek, M. Reufer, L. G. Wilson, A. N. Morozov, and W. C. K. Poon, Proc. Natl. Acad. Sci. U.S.A. 111, 17771 (2014).

[26] A. Wittmeier, A. Leeth Holterhoff, J. Johnson, and J. G. Gibbs, Langmuir 31, 10402 (2015).

[27] C. Kurzthaler, S. Leitmann, and T. Franosch, Sci. Rep. 6, 36702 (2016).

[28] J. Rudnick and G. Gaspari, Elements of the Random Walk: An Introduction for Advanced Students and Researchers (Cambridge University Press, Cambridge, England, 2004).

[29] K. Martens, L. Angelani, R. Di Leonardo, and L. Bocquet, Eur. Phys. J. E 35, 84 (2012).

[30] A. T. Brown, I. D. Vladescu, A. Dawson, T. Vissers, J. Schwarz-Linek, J. S. Lintuvuori, and W. C. K. Poon, Soft Matter 12, 131 (2016).

[31] S. Ebbens, D. Gregory, G. Dunderdale, J. Howse, Y. Ibrahim, T. Liverpool, and R. Golestanian, Europhys. Lett. 106, 58003 (2014).

[32] A. T. Brown, W. C. K. Poon, C. Holm, and J. de Graaf, Soft Matter 13, 1200 (2017).

[33] A. I. Campbell and S. J. Ebbens, Langmuir 29, 14066 (2013).

[34] K. Dietrich, D. Renggli, M. Zanini, G. Volpe, I. Buttinoni, and L. Isa, New J. Phys. 19, 065008 (2017).

[35] A. Edelstein, N. Amodaj, K. Hoover, R. Vale, and N. Stuurman, Computer control of microscopes using $\mu$ manager, in Current Protocols in Molecular Biology (John Wiley and Sons, New York, 2010).

[36] See Supplemental Material at http://link.aps.org/ supplemental/10.1103/PhysRevLett.121.078001 for further details regarding the theoretical calculations and the data analysis, which includes Refs. [37-44].

[37] B. ten Hagen, S. van Teeffelen, and H. Löwen, J. Phys. Condens. Matter 23, 194119 (2011).

[38] C. Gardiner, Stochastic Methods: A Handbook for the Natural and Social Sciences, Springer Series in Synergetics (Springer, Berlin, Heidelberg, 2009).

[39] C. Kurzthaler and T. Franosch, Phys. Rev. E95, 052501 (2017).

[40] DLMF, NIST Digital Library of Mathematical Functions, http://dlmf.nist.gov/, Release 1.0.10 of 2015-08-07, online companion to Ref. [41].

[41] NIST Handbook of Mathematical Functions, edited by F. W. J. Olver, D. W. Lozier, R. F. Boisvert, and C. W. Clark (Cambridge University Press, New York, NY, 2010), print companion to Ref. [40].

[42] R. H. Byrd, P. Lu, J. Nocedal, and C. Zhu, SIAM J. Sci. Comput. 16, 1190 (1995).

[43] B. Efron, Biometrika 68, 589 (1981).

[44] T. Savin and P. S. Doyle, Phys. Rev. E 71, 041106 (2005).

[45] F. J. Sevilla and M. Sandoval, Phys. Rev. E 91, 052150 (2015).

[46] C. H. Ziener, M. Rückl, T. Kampf, W. R. Bauer, and H. P. Schlemmer, J. Comput. Appl. Math. 236, 4513 (2012).

[47] S. Ebbens, R. A. L. Jones, A. J. Ryan, R. Golestanian, and J. R. Howse, Phys. Rev. E 82, 015304 (2010).

[48] F. Giavazzi, P. Edera, P. J. Lu, and R. Cerbino, Eur. Phys. J. E 40, 97 (2017).
[49] M. K. Phibbs and P. A. Giguère, Can. J. Chem. 29, 173 (1951).

[50] Edited by, W. M. Haynes, CRC Handbook of Chemistry and Physics, 93rd ed. (CRC Press, Boca Raton, FL, 2013).

[51] J. Happel and H. Brenner, Low Reynolds Number Hydrodynamics: With Special Applications to Particulate Media (Springer Science \& Business Media, New York, 2012), Vol. 1.

[52] J. Leach, H. Mushfique, S. Keen, R. Di Leonardo, G. Ruocco, J. M. Cooper, and M. J. Padgett, Phys. Rev. E 79, 026301 (2009).

[53] S. Ebbens, M.-H. Tu, J. R. Howse, and R. Golestanian, Phys. Rev. E 85, 020401 (2012).

[54] X. Zheng, B. ten Hagen, A. Kaiser, M. Wu, H. Cui, Z. Silber-Li, and H. Löwen, Phys. Rev. E 88, 032304 (2013).

[55] R. Golestanian, T. B. Liverpool, and A. Ajdari, Phys. Rev. Lett. 94, 220801 (2005).

[56] M. N. Popescu, W. E. Uspal, and S. Dietrich, Eur. Phys. J. Spec. Top. 225, 2189 (2016).

[57] R. Golestanian, T. B. Liverpool, and A. Ajdari, New J. Phys. 9, 126 (2007).

[58] C. Kurzthaler and T. Franosch, Soft Matter 13, 6396 (2017).

[59] F. Kümmel, B. ten Hagen, R. Wittkowski, I. Buttinoni, R. Eichhorn, G. Volpe, H. Löwen, and C. Bechinger, Phys. Rev. Lett. 110, 198302 (2013).

[60] B. ten Hagen, F. Kümmel, R. Wittkowski, D. Takagi, H. Löwen, and C. Bechinger, Nat. Commun. 5, 4829 (2014).

[61] E. Lauga, W. R. DiLuzio, G. M. Whitesides, and H. A. Stone, Biophys. J. 90, 400 (2006).

[62] R. Di Leonardo, D. Dell'Arciprete, L. Angelani, and V. Iebba, Phys. Rev. Lett. 106, 038101 (2011).

[63] P. Romanczuk and L. Schimansky-Geier, Phys. Rev. Lett. 106, 230601 (2011).

[64] J. S. Lintuvuori, A. T. Brown, K. Stratford, and D. Marenduzzo, Soft Matter 12, 7959 (2016).

[65] Z. Shen, A. Würger, and J. S. Lintuvuori, Eur. Phys. J. E 41, 39 (2018).

[66] J. Palacci, C. Cottin-Bizonne, C. Ybert, and L. Bocquet, Phys. Rev. Lett. 105, 088304 (2010).

[67] I. Theurkauff, C. Cottin-Bizonne, J. Palacci, C. Ybert, and L. Bocquet, Phys. Rev. Lett. 108, 268303 (2012).

[68] J. Palacci, S. Sacanna, A. P. Steinberg, D. J. Pine, and P. M. Chaikin, Science 339, 936 (2013).

[69] M. Paoluzzi, R. Di Leonardo, and L. Angelani, J. Phys. Condens. Matter 26, 375101 (2014).

[70] X. Ma, K. Hahn, and S. Sanchez, J. Am. Chem. Soc. 137, 4976 (2015).

[71] S. Sengupta, K. K. Dey, H. S. Muddana, T. Tabouillot, M. E. Ibele, P. J. Butler, and A. Sen, J. Am. Chem. Soc. 135, 1406 (2013).

[72] C. Riedel, R. Gabizon, C. A. Wilson, K. Hamadani, K. Tsekouras, S. Marqusee, S. Pressé, and C. Bustamante, Nature (London) 517, 227 (2015).

[73] A. Joseph, C. Contini, D. Cecchin, S. Nyberg, L. Ruiz-Perez, J. Gaitzsch, G. Fullstone, X. Tian, J. Azizi, J. Preston, G. Volpe, and G. Battaglia, Sci. Adv. 3, e1700362 (2017).

[74] C. Kurzthaler, C. Devailly, J. Arlt, T. Franosch, W. Poon, V. Martinez, and A. Brown, Part 1: http://dx.doi.org/10.7488/ds/ 2373; Part 2: http://dx.doi.org/10.7488/ds/2374, [dataset], (2018). 